\title{
Variation in the Magneto-Impedance (MI) Effect According to the Shape of Patterned $\mathrm{Co}_{30} \mathrm{Fe}_{34} \mathrm{Ni}_{36}$ Alloys
}

\author{
Hyun Kyung Kim¹, Do Hun Kim¹, De Rac Son ${ }^{2}$, and Won Young Jeung ${ }^{1 *}$ \\ ${ }^{1}$ Division of Materials Science \& Engineering, Korea Institute of Science and Technology (KIST), 39-1 Haweolgok-dong, \\ Seongbuk-gu, Seoul 136-791, Korea \\ ${ }^{2}$ Applied Optics and Electromagnetics, Hannam University, Ojung-dong 133, Daeduk-gu, Daejeon 306-791, Korea
}

(Received 7 March 2008)

\begin{abstract}
The magneto impedance (MI) behaviors of patterned $\mathrm{Co}_{30} \mathrm{Fe}_{34} \mathrm{Ni}_{36}$ microwire were investigated with respect to its shape variation. After preparing $\mathrm{Co}_{30} \mathrm{Fe}_{34} \mathrm{Ni}_{36}$ microwires using electrodeposition and photolithography methods, impedance measurements were conducted to compare the MI ratios of the devices with different aspect ratios. As a result, the anisotropy field and transverse permeability were found to be strongly affected by the aspect ratio of the device. The external field value at the maximum impedance and maximum sensitivity of the device was found to increase with increasing device width, which was attributed to the increased transverse anisotropy with decreasing aspect ratio. While an increase in the thickness also contributed to an increase in the MI ratio, a variation in the thickness not only increased the anisotropic field, but the variation in the MI ratio was as also affected by the skin effect. Conversely, the MI ratios of the present devices were hardly affected by variations in the length. Considering the typical aspect ratios of our devices, it was expected that the length effect would emerge when the aspect ratio was reduced to less than 10. Nevertheless, our results show that for the practical application of MI devices, the MI characteristics can be optimized by tailoring the aspect ratio of the devices.
\end{abstract}

Keywords : magneto impedance (MI), anisotropy field, skin effect, permeability

\section{Introduction}

When an external magnetic field is applied to soft magnetic materials using an applied ac current, the impedance of the material sensitively changes with the magnetic field. This effect, called the magneto impedance (MI) effect, is one of the most promising electromagnetic phenomena of soft magnetic materials, which is believed to have potential applications, such as highly sensible magnetic sensors and magnetic recording heads [1-4]. This effect has been found to be appropriate for application to sensors based on the sensitive MI response reported for Co-based amorphous wires, with almost zero magnetostriction [57].

For practical applications, the miniaturized devices require the smallest possible MI elements. However, only a few investigations have been devoted toward the effects of the size and shape of these devices $[8,9]$. In addition,

*Corresponding author: Tel: +82-2-958-5422

Fax: +82-2-958-6839, e-mail: wyjeung@kist.re.kr the MI effect of crystalline micro patterned magnetic materials, as well as its dependence on the material shape and dimension, remains to be fully understood.

Therefore, the relationship between the device shape and the MI ratio were investigated using micro patterned nanocrystalline CoFeNi alloys. By precisely optimizing the MI properties, by tailoring the aspect ratios, the ultra high sensitivity of MI sensors could be exploited for actual applications.

\section{Experiment}

For the electrodeposition process, CoFeNi soft magnetic alloys were sputtered onto a Si wafer. In our previous work [10-13], the various effects of the electroplating conditions on the soft magnetic property of CoFeNi alloys were examined. The optimal composition was found to be $\mathrm{Co}_{30} \mathrm{Fe}_{34} \mathrm{Ni}_{36}$, with a saturation magnetic-flux density $\left(\mathrm{B}_{\mathrm{s}}\right)$, permeability $(\mu)$ and coercivity field $\left(\mathrm{H}_{\mathrm{c}}\right)$ of $1.9 \mathrm{~T}, 3.4 \times$ $10^{6}$ and $12 \mathrm{~A} / \mathrm{m}$, respectively. In addition, the transverse uniaxial magnetic anisotropy of the electroplated $\mathrm{CoFeNi}$ 


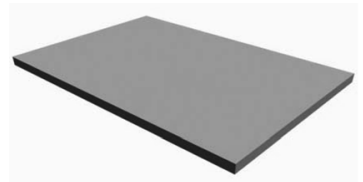

(a) Electroplating of $\mathrm{CoFeNi}$ alloys

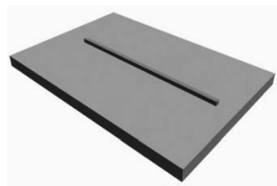

(b) PR patterning

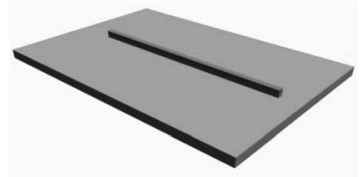

(c) Wet etching

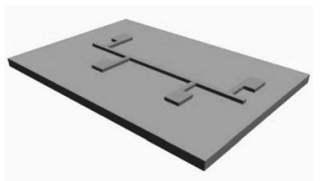

(d) Au pad deposition

Fig. 1. Fabrication process for the patterned MI device using CoFeNi microwire.

alloys was confirmed using hysteresis loop measurements. Using these conditions, a soft magnetic alloy with thickness of $1 \mu \mathrm{m}$ was electroplated onto a sputtered $\mathrm{CoFeNi} /$ Ti/Si wafer $\left(1.5 \times 15 \mathrm{~mm}^{2}\right)$. Fig. 1 shows the fabrication process for the MI device made of micro patterned CoFeNi alloys. To investigate the MI characteristics with respect to the device shape, microwires of various dimensions (from $30 \times 1 \times 1500 \mu \mathrm{m}$ to $150 \times 10 \times 7500 \mu \mathrm{m}$ (width $\times$ thickness $\times$ length)) were patterned by photolithography, with subsequent wet etching. Au electrodes were deposited by magnetron sputtering and used for four probe measurement.

The magnetic properties were determined using a vibrating sample magnetometer (VSM, Lake Shore 7400). For the impedance measurement, a function generator was used as the ac signal source, while the root mean square (RMS) value of the device ac voltage was converted to dc using an AD8361 RF detector chip, which was then amplified to increase the signal to noise ratio.

\section{Results and Discussion}

The operation of the MI effect is classified into three ranges depending on frequency of the exciting ac signal. First, below the $\mathrm{MHz}$ region, the variation in the inductive reactance dominates the total impedance changes under the applied magnetic field, which is termed the magneto inductive effect. In this regime, due to the low frequency, the applied ac signal can be distributed over almost the entire cross section, with the material's magnetization largely dominated by domain wall motion. The resultant impedance profile shows one peak at zero magnetic field, as well as monotonic decreases in the impedance with increases in the magnetic field. Secondly, when the exciting frequency exceeds a few $\mathrm{MHz}$, a severe eddy current inhibits the domain wall motion, where the magnetization rotation determines the magnetization process. Also, due to the skin effect, the applied electromagnetic field is concentrated at the surface of the material. This increased surface contribution greatly modifies the impedance profile, with increases in the variation well above a few tens of a percentage. Finally, in the microwave regime above $1 \mathrm{GHz}$, ferromagnetic resonance (FMR) contributes to the GMI profile. As our operating frequency was between 4 and $20 \mathrm{MHz}$, it was expected that the GMI profile of the present devices stemmed from the skin effect and the response of the transverse magnetic permeability during magnetization rotation.

\subsection{Width effect}

Fig. 2 illustrates the field dependence of the MI behavior with variations in the width of the $\mathrm{CoFeNi}$ microwire at $20 \mathrm{MHz}$. The length and thickness of the device were fixed at 7500 and $1 \mu \mathrm{m}$, respectively. Typical symmetric behavior and double-peak patterns were clearly shown, which was consistent with conventional MI devices in an RF frequency. The external field values of the devices at the maximum impedance were found to be largely dependant on the device width. Stating from $650 \mathrm{~A} / \mathrm{m}(8 \mathrm{Oe})$ of the peak field, with a device width of $30 \mu \mathrm{m}$, which increased to $1140 \mathrm{~A} / \mathrm{m}(15 \mathrm{Oe})$ with a width of $150 \mu \mathrm{m}$. The observed width effect can be attributed to the increase in the transverse anisotropy field $\left(\mathrm{H}_{\mathrm{k}}\right)$. In the RF frequency regime, the impedance is well know to reach a maximum when the external field is approximately equal to that of the transverse anisotropy field [14, 15] Therefore, the tendency of the magnetic field to increase at the

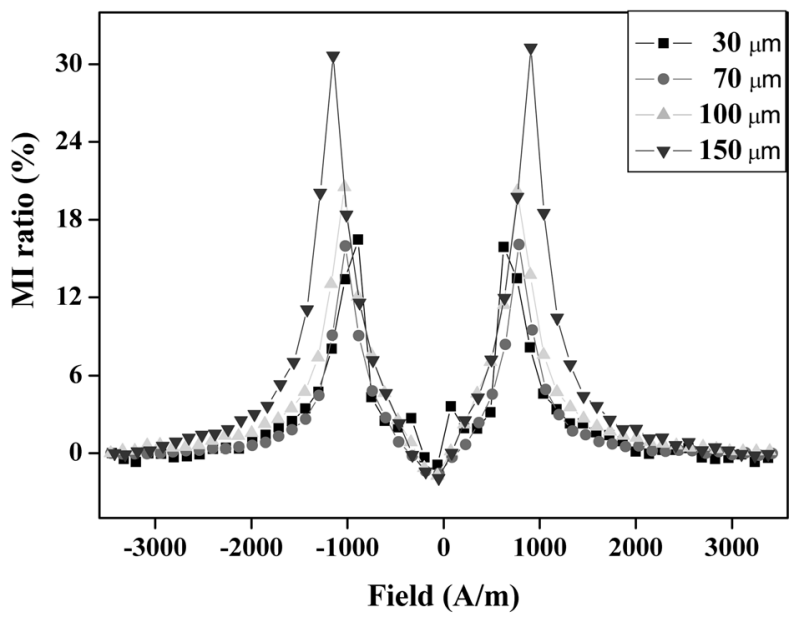

Fig. 2. MI behavior as a function of the CoFeNi microwire width. 


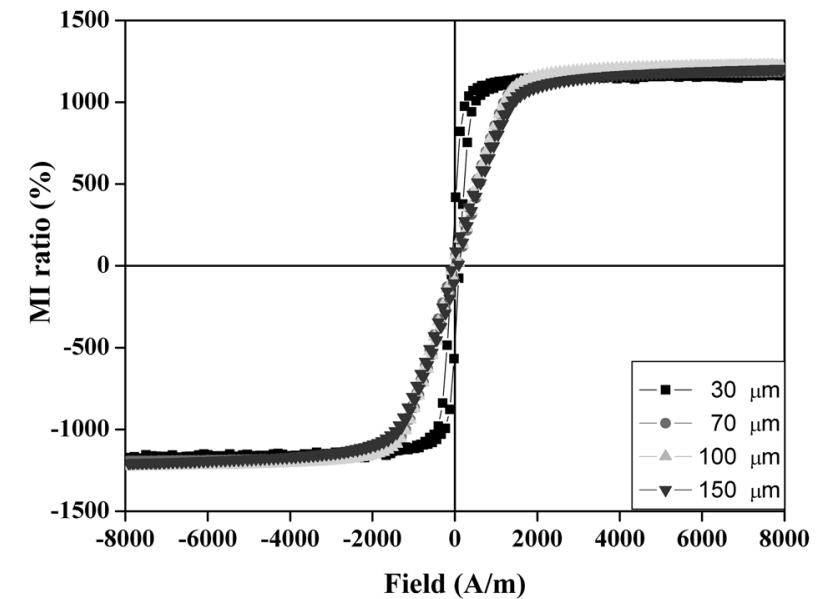

Fig. 3. Hysteresis loop with variation of the CoFeNi microwire width.

maximum impedance can be verified by measuring the transverse anisotropy field using a VSM. As shown in Fig. 3, the device transverse anisotropy gradually increased from about 480 (6 Oe) to $1120 \mathrm{~A} / \mathrm{m}(15 \mathrm{Oe})$ with decreasing device width due to the reducing of the shape of anisotropy. Fig. 2 shows that the maximum sensitivity of the devices increases with increasing device width. In addition, it can be seen that the variation in the MI ratio with respect to changes in the width from 15 to $30 \%$ for device widths of 30 and $150 \mu \mathrm{m}$, respectively. From previous work, the impedance maximum was reported to gradually broaden with increases in the peak field point, which was consistent with our results $[16,17]$.

\subsection{Thickness effect}

The thickness dependence of the MI ratio was investigated by fixing the width and length of the device at 150 and $7500 \mu \mathrm{m}$, respectively, as shown in Fig. 4. As the thickness was increased from 1 to $10 \mu \mathrm{m}$, the MI ratio increased from 30 to $180 \%$ at $20 \mathrm{MHz}$. In the RF frequency regime (1 KHz-100 MHz), the MI ratio was significantly affected by the skin depth of the material, and the ratio between the skin depth and device thickness was a crucial factor in determining the MI effect $[18,19]$. From classical electrodynamics, the skin depth of the material is given by,

$$
\delta=\frac{1}{\sqrt{\pi f \sigma \mu}}
$$

where the frequency of current is $f, \sigma$ is the device conductivity and $\mu$ is the transverse permeability of the device. Using the values of current MI device, the skin depth was estimated to be $0.81 \mu \mathrm{m}$ when the $f, \Omega$ and $\mu$ were $20 \mathrm{MHz}, 9.8 \Omega$ and $3.4 \times 10^{6}$, respectively. There-

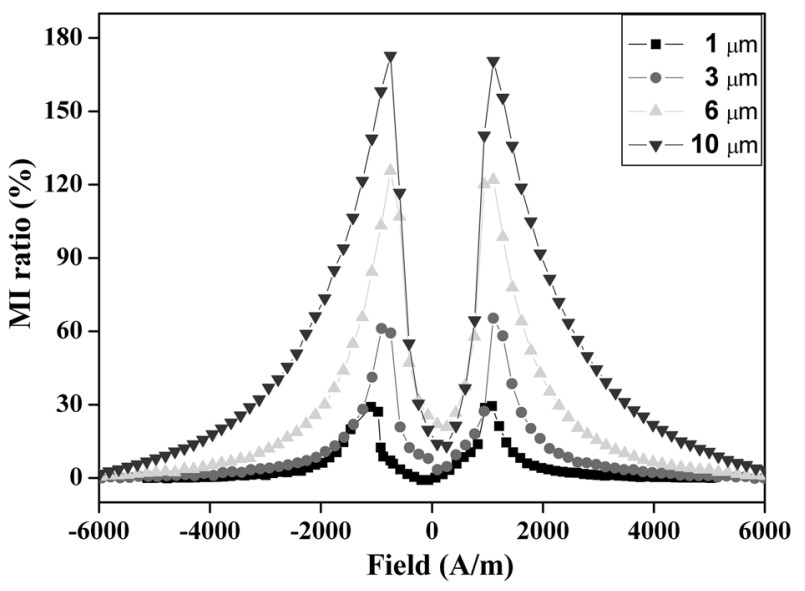

Fig. 4. Impedance changes as a function of the CoFeNi microwire thickness.

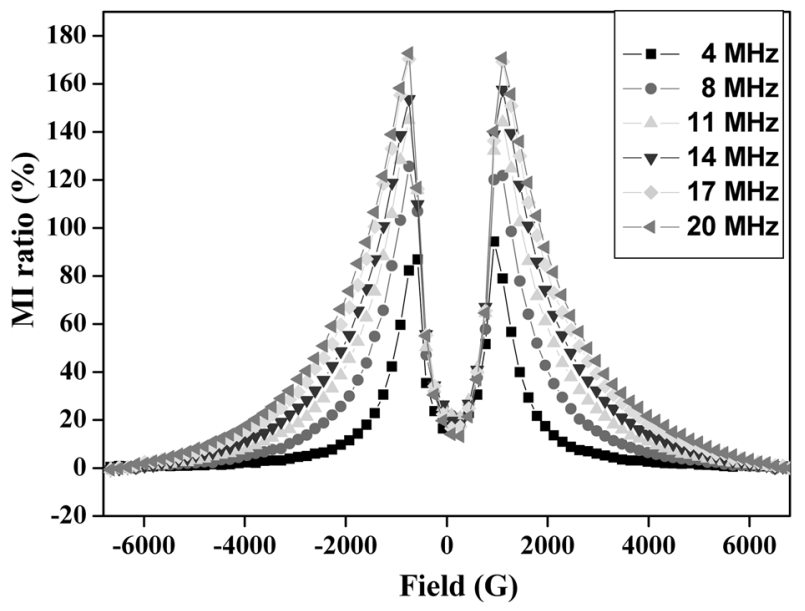

Fig. 5. Frequency dependence of the MI ratio of the CoFeNi microwire.

fore, as the thickness of the device increased, the skin depth to thickness ratio decreased. In order to further examine the relationship between skin depth and MI ratio, a device with dimension fixed at $150 \times 10 \times 7500 \mu \mathrm{m}$ (width $\times$ thickness $\times$ length) was used to investigate the change in the MI ratio at frequencies ranging from 4 to 20 MHz. As illustrated in Fig. 5, with increasing frequency, hence decreasing skin depth, resulted in an increase in the MI ratio from 100 to $180 \%$, and shows that as the skin depth decreases, the ratio of the skin depth to the device thickness decreases, leading to an increase in the MI ratio.

The relationship between the skin depth and thickness, as well as the change in the transverse anisotropy with device thickness affected the MI profile. As shown in Fig. 4 , as the external magnetic field value at maximum impedance was found to increase from about 1000 to $1120 \mathrm{~A} / \mathrm{m}$. Therefore, from the above discussion, the transverse anisotropy, although not as significantly as the 


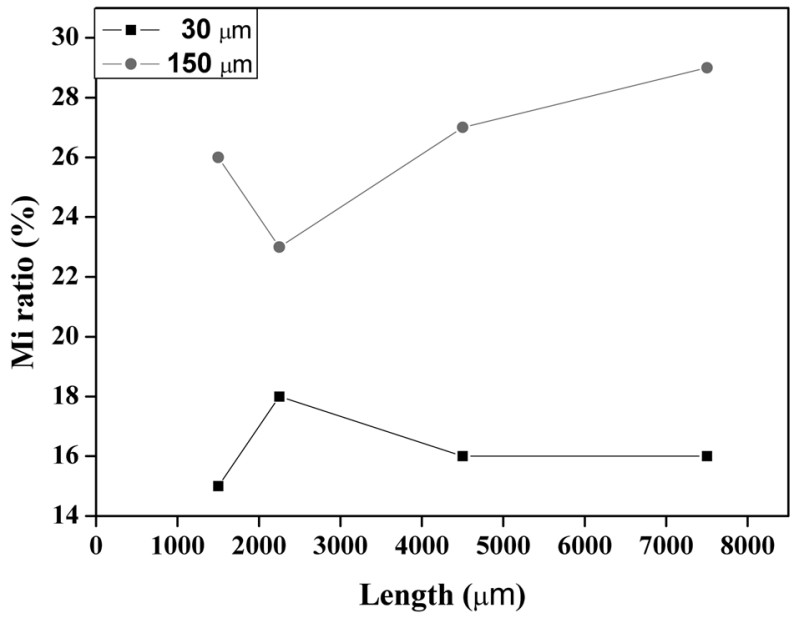

Fig. 6. Impedance variation of the CoFeNi microwire as a function of the length.

width dependence, was increased; hence, this increase in the transverse anisotropy contributes to the enhanced MI ratio. Consequently, the dependence of the MI ratio with variations in the thickness results from the two effects, these being the variation in the skin depth to thickness ratio, and the variation in the transverse anisotropic field.

\subsection{Length effect}

Fig. 6 shows the change in the MI ratio with respect to variations in the length for device widths of 30 and 150 $\mu \mathrm{m}$ and a thickness fixed at $1 \mu \mathrm{m}$. On varying the device length, the aspect ratio (L/W) changed from 10 to 250 at $20 \mathrm{MHz}$. However, the MI behavior found to show only slight dependence on the device length. Generally, as the sample length increases at a fixed width, so does the aspect ratio, which decreases the demagnetizing factor along the wire axis; however, that along the transverse axis increases. Therefore, a well defined easy axis is induced in the longitudinal direction due to the shape of the anisotropy. However, in our previous experiments, because the minimum aspect ratio of the current devices exceeded 10, the demagnetizing field seemed to hardly change with respect to the sample length. Therefore, although there was a possibility that the MI ratio can be affected by variation in the demagnetizing field induced by a change in length, no substantial dependence of the MI ratio on the device length was observed. Further work on the micron-scaled MI devices, where the typical aspect ratio was reduced to 3 , has been presented elsewhere [20]. Nevertheless, from the length dependent MI ratio measurement, to induce a significant change in the MI ratio with respect to the device length, the estimated aspect ratio between the length and width should be reduced to less than 10 .

\section{Conclusion}

In summary, the MI behaviors have been investigated with respect to variation in the shape of a nanocrystalline $\mathrm{CoFeNi}$ microwire. The MI behavior was found to be largely dependent on the variations in the device width and thickness, but not on the device length. By varying the device width, the value of the magnetic field increased at the maximum impedance from 650 to $1140 \mathrm{~A} / \mathrm{m}$. Moreover, the MI ratio also found to vary with changes in the width and when the maximum MI ratio and device width were changed from 15 to $30 \%$ and from 30 to 150 $\mu \mathrm{m}$, respectively. Furthermore, as the thickness was increased from 1 to $10 \mu \mathrm{m}$, the MI ratio increased from 30 to $180 \%$. Finally, the lack of dependence of the MI ratio on the device length was attributed to the relatively large minimum aspect ratio of our devices (over 10).

\section{Acknowledgment}

This work was supported by a grant from the Fundamental R\&D Program for Core Technology of Materials, funded by the Ministry of Commerce, and the R\&D Program for NT-IT Fusion Strategy of Advanced Technologies, Industry and Energy, Republic of Korea.

\section{References}

[1] K. Mohri, K. Kawashima, T. Kohzawa, and Y. Yoshida, IEEE Trans. Magn. 29, 1245 (1993).

[2] S. H. Park, D. W. Chun, J. H. Han, Y. H. Kim, and W. Y. Jeung, Phys. Stat. Sol. (a). 204, 4071 (2002).

[3] H. B. Lee, Y. S. Kim, and S. C. Yu, J. Magnetics 7(4), 160 (2002).

[4] A. T. Le, N. D. Ha, C. O. Kim, J. R. Rhee, N. Chau, N. Q. Hoa, N. D. Tho, and H. B. Lee, J. Magnetics 11(1), 55 (2006).

[5] T. Mizoguchi and G. S. Cargill III, J. Appl. Phys. 50, 3570 (1579).

[6] C. Dong, S. Chen, and T. Y. Hsu, J. Magn. Magn. Mater. 250, 288 (2002).

[7] V. Satya Narayana Murthy, S. Venkatesh, and G. Makandeyulu, J. Appl. Phys. 99, 08F108 (2006).

[8] M. Vaquez, Y.-F. Li, and D.-X. Chen, J. Appl. Phys. 91, 6539 (2002).

[9] G. V. Kurlyandkaya, J. M. Barandiaran, M. Vaquez, D. Garcia, and N. V. Dmitrieva, J. Magn. Magn. Mater. 740, 215-216 (2000).

[10] W. Y. Jeung, H. K. Kim, and J. O. Lee, J. Kor. Mag. Soc., 15(4), 241 (2005).

[11] J. O. Lee, H. K. Kim, G. H. Kim, and W. Y. Jeung, J. Appl. Phys. 99, 08 B704 (2006).

[12] W. Y. Jeung, H. K. Kim, and C. B. Park, J. Kor. Mag. 
Soc., 16(5), 249 (2006)

[13] H. K Kim, D. W. Chun, J. H. Han, K. B. Kim, and W. Y. Jeung, Phys. Stat. Sol. (a). 204, 4104 (2007).

[14] D. de Cos, A. Garcia-Arribas, and J. M. Barandiaran, IEEE Trans. Magn. 41, 3649 (2005).

[15] S. S. Yoon, C. G. Kim, J. Jang, and H. Lee, IEEE Trans. Magn. 36, 2872 (2000).

[16] H. Chiriac, T. A. Óvári, and C. S. Marinescu, J. Appl. Phys. 83, 6584 (1998).
[17] C. Garcia, J. Gonzalez, A. Chizhik, A. Zhukov, and J. M. Blanco, J. Appl. Phys. 95, 6756 (2004).

[18] H. B. Nie, A. B. Pakhomov, X. Yan, X. X. Zhang, and M. Knobel, Sol. Stat. Commun. 112, 285 (1999).

[19] P. Jantaratana and C. Sirisathitkul, IEEE Trans. Magn. 42, 358 (2006).

[20] D. H. Kim, H. K. Kim, S. H. Park, and W. Y. Jeung, Submit to IEEE Trans. Magn. 